\title{
Equation of state and intermolecular interactions in fluid hydrogen from Brillouin scattering at high pressures and temperatures
}

\author{
Kiyoto Matsuishia) \\ Institute of Materials Science, University of Tsukuba, Tsukuba, Ibaraki 305-8573, Japan \\ Eugene Gregoryanz, Ho-kwang Mao, and Russell J. Hemley \\ Geophysical Laboratory, Carnegie Institution of Washington, Washington, D.C. 20015
}

(Received 13 June 2002; accepted 21 March 2003)

\begin{abstract}
Brillouin scattering spectra of fluid hydrogen were measured at high pressures (1 to $13 \mathrm{GPa}$ ) and temperatures ( 293 to $526 \mathrm{~K}$ ). From these sound velocity data together with previously reported volume and ultrasonic velocity data at low pressures and temperatures, we determined a Benedict-type $P-V-T$ equation of state valid for fluid hydrogen up to the maximum pressures and temperatures of this study with an average deviation of $1.0 \%$ from the new and previously published experimental data. Using the equation of state, the pressure and temperature dependences of thermodynamic properties were calculated. We examined three types of intermolecular potentials for fluid hydrogen, and found that the Hemley-Silvera-Goldman potential gives superior fits to the experimentally derived equation of state over a wide temperature range above $6 \mathrm{GPa}$. Discrepancies found in the high temperature range at low pressures provide additional constraints on determination of the intermolecular potential. (C) 2003 American Institute of Physics. [DOI: 10.1063/1.1575196]
\end{abstract}

\section{INTRODUCTION}

The behavior of hydrogen over a broad range of thermodynamic conditions is fundamental to a variety range of problems in the physical sciences. ${ }^{1-4}$ Of paramount importance is the accurate determination of the pressure-volumetemperature $(P-V-T)$ equation of state (EOS) in its fluid and solid phases as well as the melting line that defines the phase boundary between these phases. At a fundamental level, EOS data provide key information on the evolution of intermolecular and interatomic interactions with changing pressure and temperature. Indeed, the construction of accurate intermolecular and interatomic potentials from such data is exceedingly useful for representing both the microscopic and bulk properties of the material over a broad $P-T$ range. Measurements of the EOS of the fluid are also essential for developing a complete thermodynamic description for hydrogen for thermochemical calculations (e.g., Ref. 5). There is also growing interest in the EOS of the high $P-T$ fluid in view of the reported transitions at high pressures on shock compression (starting at $50 \mathrm{GPa}$ ). ${ }^{6-10}$ The behavior of fluid hydrogen under these conditions is crucial for models of planetary interiors. ${ }^{11}$

The EOS of the room-temperature solid has been determined to high accuracy by diffraction techniques to maximum pressures of $120 \mathrm{GPa} .{ }^{12-17}$ However, less attention has been given to the fluid in static compression experiments. In contrast, shock-compression EOS studies have probed the fluid Hugoniot to pressures in the 100-GPa range but at very high temperatures (e.g., reaching $>10000 \mathrm{~K}$ at these pressures). Early static pressure EOS studies on the fluid were carried out using a differential manometer ${ }^{18}$ and volumetric techniques ${ }^{19,20}$ to maximum pressures of a few gigapascals.

${ }^{a)}$ Electronic mail: kiyoto@bk.tsukuba.ac.jp
As an alternative to volumetric or diffraction methods, measurement of thermodynamic properties that depend on derivatives of $P, V$, and $T$ (e.g., sound velocity), provide a direct determination of the EOS and are especially applicable to the fluid. Mills and co-workers ${ }^{20}$ investigated the EOS of fluid hydrogen by ultrasonic and volumetric techniques at $P \leqslant 2 \mathrm{GPa}$ and $T \leqslant 307 \mathrm{~K}$, and fit the data to a Benedict-type function. The EOS of the fluid was subsequently examined by Brillouin scattering measurements of the sound velocity as a function of pressure to $5.35 \mathrm{GPa}^{21,22} \mathrm{~A}$ significant deviation between the extrapolated Mills et al. roomtemperature EOS was found. ${ }^{21}$ The accuracy of the Brillouin data was subsequently questioned because of possible reactions of the gasket material with fluid hydrogen. ${ }^{23}$ Additional Brillouin measurements were reported by Pratesi et al., ${ }^{22}$ who derived a different functional form for the fluid hydrogen density up to $5.35 \mathrm{GPa}$ at room temperature.

These EOS determinations have provided a basis for a variety of calculations and interpretations of phenomena at higher pressures and temperatures. The results were used with a Mie-Grüneisen model for pressures up to $100 \mathrm{GPa}$ and temperatures between 100 and $1000 \mathrm{~K}^{5}$ Although the authors claimed that all experimental data were reproduced within $0.5 \%$, the data available at that time were limited in $P$ - $T$ range (for example, only below $2 \mathrm{GPa}$ for fluid hydrogen). ${ }^{5}$ In addition, they used a modified van der Waals equation (i.e., a correction to the ideal gas) for the EOS of the fluid to simplify extrapolations to high $P-T$ conditions where no experimental data were available. Also, effective pair potentials have been calculated to fit shock-wave data to $70 \mathrm{GPa}^{24,25}$ These potentials, including a modification to pair potentials originally fit to low pressure EOS data for the solid (to $<2.5 \mathrm{GPa}$ ) ${ }^{26}$ required additional adjustment to fit accurate $\mathrm{x}$-ray diffraction data obtained to higher pressure. ${ }^{14}$ 
This type of effective potential was also used to examine reported seismic observations for Jupiter, which in principle contain a wealth of information on the interior structure of the planet. ${ }^{27-29}$ By combining data from synchrotron $\mathrm{x}$-ray diffraction and Brillouin scattering for the solid, ${ }^{30}$ a new effective pair potential for solid hydrogen was determined. ${ }^{27}$ This function also reproduced independent high $P-T$ shock wave data. Using the effective pair potential, the sound velocity was extrapolated to the high-temperature fluid at Jovian interior conditions to place constraints on models for the planet. $^{27,28}$ However, an effective pair potential derived from high-temperature data for the fluid phase is naturally preferred over that obtained from analysis of low-temperature solid phase data in order to develop an accurate description of the hot dense fluid.

Accurate EOS data for the fluid also provide an important baseline for understanding still higher pressure behavior. Numerous theoretical studies of hydrogen at more extreme pressures have predicted anomalous behavior in thermodynamic properties of the high density fluid (Refs. 9 and 31 and references therein). A shock-induced transformation into a conducting fluid has been reported for hydrogen ${ }^{7}$ and deuterium. ${ }^{8,10}$ The need for accurate high-pressure EOS models for hydrogen has been revived in order to understand these results. In addition, Hugoniot data have been reported from gas gun, ${ }^{6,32}$ laser shock, ${ }^{8,33,34}$ and most recently magnetic drives. ${ }^{35}$ However, discrepancies among these results have been the subject of current controversy. ${ }^{8,33-40}$ Examination of the $P-V-T$ EOS of the fluid by static compression techniques beginning in the low $P-T$ range may shed light on this problem. Moreover, measurements at these conditions are necessary for an accurate determination of the initial conditions in shock experiments on precompressed samples. ${ }^{41}$

Static high $P-T$ studies of hydrogen needed to address these issues have proven difficult, primarily because of problems associated with containing the material in high-pressure devices under these conditions. The melting curve of hydrogen has been reported on the basis of visual observation in a diamond-cell to $7.8 \mathrm{GPa}$ and $373 \mathrm{~K} .{ }^{42,43}$ More recently, Datchi et al. $^{44}$ extended the measurement of the melting curve to $15 \mathrm{GPa}$ using $P-T$ scan techniques. Here we report Brillouin scattering measurements of fluid and solid hydrogen $\left(n-\mathrm{H}_{2}\right)$ at high pressures from 1 to $13 \mathrm{GPa}$ and temperatures from 293 to $526 \mathrm{~K}$. The pressure dependence of melting was directly observed by Brillouin scattering. Compressional sound velocity data for the fluid were used to determine a Benedicttype EOS applicable for this phase in the measured $P-T$ range. The temperature and pressure dependences of the thermodynamic properties were then derived from the EOS. We examine the intermolecular potentials proposed previously for hydrogen and discuss their validity for the fluid phase in this high $P-T$ range. We focus our attention on the fluid phase of hydrogen, preliminary results have been reported. ${ }^{45}$ Brillouin measurements for solid hydrogen will be presented elsewhere together with complementary Raman data in order to relate the elastic and vibrational properties for solid and fluid hydrogen at these $P-T$ conditions.
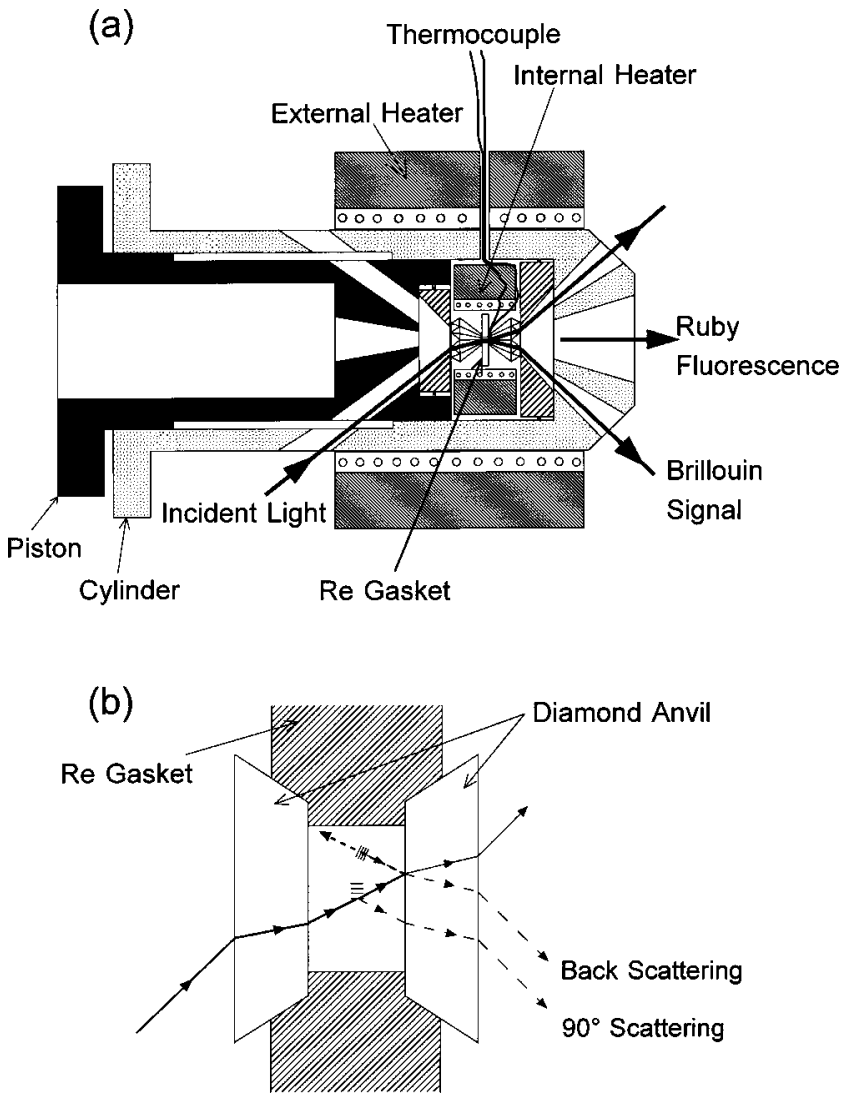

FIG. 1. (a) Schematic view of the experimental setup of the high temperature diamond anvil cell in the Brillouin scattering configuration. (b) Scattering geometry in the sample chamber in an enlarged view (solid line, incident light; short-dashed line, reflected light; long-dashed line, Brillouin scattering signals).

\section{EXPERIMENT}

Brillouin scattering experiments were carried out in an internally and externally heated Mao-Bell diamond-anvil cell mounted with $600 \mu \mathrm{m}$ culet diamonds. Schematic views of the heated diamond-anvil cell and the Brillouin scattering geometry are shown in Figs. 1(a) and 1(b), respectively. A donut shaped furnace $(20 \mathrm{~mm}$ in outer-diameter, $4 \mathrm{~mm}$ thickness) with a Pt-wire resistance heater ${ }^{46}$ was mounted around the diamond on the piston of the cell. Temperatures were measured with a $\mathrm{Pt}-\mathrm{Pt} / 10 \% \mathrm{Rh}$ thermocouple placed very close to the sample chamber $(150 \mu \mathrm{m}$ diameter, $70 \mu \mathrm{m}$ thickness) in preindented rhenium gaskets mounted between the culets of the diamonds. Temperature was also monitored by a chromel-alumel thermocouple placed externally on the back of the cylinder diamond. Both temperatures readings typically agreed to within $2 \mathrm{~K}$ below $400 \mathrm{~K}$ and to within $4 \mathrm{~K}$ at the highest temperatures, which was sufficient for this study. Obtaining this level of temperature accuracy was crucial to this study, since the pressure was calibrated using the pressure and temperature scale of the $R_{1}$ line of ruby fluorescence from a ruby sphere $(10 \mu \mathrm{m}$ in diameter) located at the edge of the gasket hole.

The temperature-induced electronic energy-level shift of the $R_{1}$ line was calculated based on a model of a two-phonon Raman process; ${ }^{47}$ 


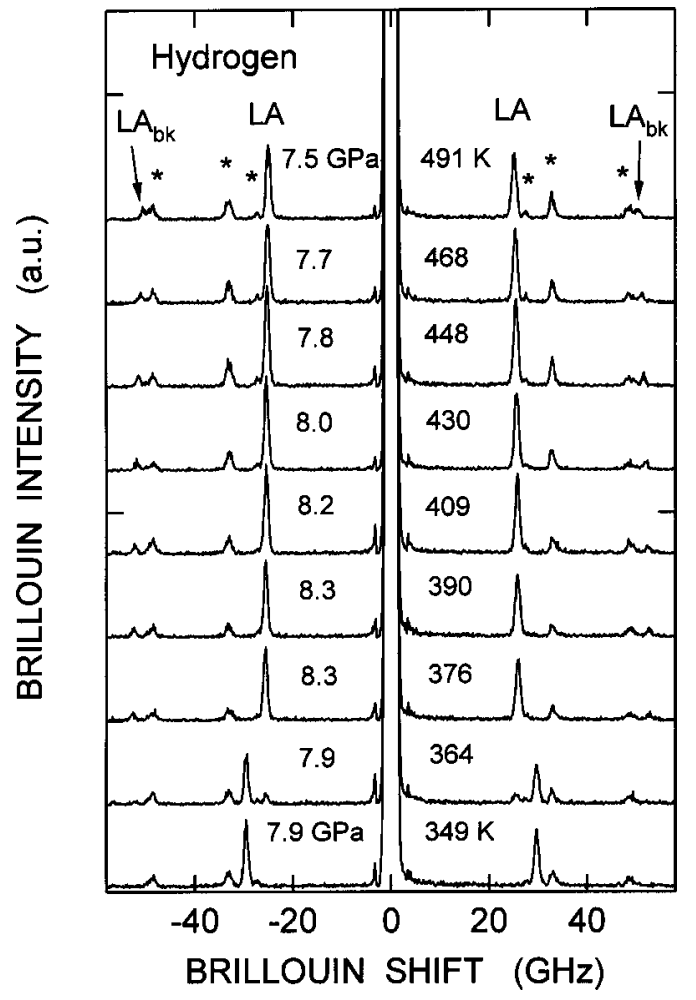

FIG. 2. Representative Brillouin scattering spectra of hydrogen from 349 to $491 \mathrm{~K}$ at $P \approx 8 \mathrm{GPa}$. The asterisks indicate Brillouin peaks from diamond. The compressional (i.e., longitudinal acoustic) modes of hydrogen on $90^{\circ}$ and $180^{\circ}$ (back-scattering) geometries are labeled LA and $\mathrm{LA}_{\mathrm{bk}}$, respectively. The central peak is the Rayleigh scattering component. Features near the base of the Rayleigh peaks are artifacts arising from the acousto-optic modulator which attenuates the laser intensity when scanning through the Rayleigh peak. The two spectra from the bottom were taken in the solid, and the others in the fluid.

$$
R_{1}(T)-R_{1}(0)=\alpha\left(\frac{T}{T_{d}}\right)^{4} \int_{0}^{T_{d} / T} \frac{x^{3}}{e^{x}-1} d x .
$$

Here $\alpha$ is the electron-phonon coupling constant, and $T_{d}$ is a Debye temperature. We applied this form for the $R_{1}$ line shift from $R_{1}(293 \mathrm{~K})$ using $\alpha=-419 \mathrm{~cm}^{-1}$ and $T_{d}=760 \mathrm{~K} .^{48} \mathrm{In}$ this formula, the direct-process terms, which derive from transitions between 1 and 2 of the ${ }^{2} E$ levels (split by $29 \mathrm{~cm}^{-1}$ at room temperature) associated with the absorption or emission of a single phonon, are neglected. This formula has been able to fit the $R_{1}$ line shift well over a wide temperature range up to $700 \mathrm{~K} .{ }^{47,49}$ Other authors have provided empirical fits for the $R_{1}$ line shift with temperature. ${ }^{50-55}$ Although the use of Eq. (1) is cumbersome, it seems to be applicable over a wider temperature range than the empirical forms. The pressure-induced shift was calibrated for quasihydrostatic conditions. ${ }^{56}$ The pressure coefficient was assumed to be temperature independent in the present $P-T$ range. ${ }^{57}$ Using the ruby pressure scale, the temperature uncertainty of $4 \mathrm{~K}$ causes the pressure uncertainty of about $0.1 \mathrm{GPa}$ at $500 \mathrm{~K}$. Since the ruby fluorescence lines of $R_{1}$ and $R_{2}$ broaden and become more difficult to resolve as temperature increases, we fitted ruby fluorescence spectra with two Lorentzian line profiles to obtain a precise $R_{1}$ peak wavelength. The cubic equation in $T$ was proposed for the $R_{1}$ shift over the temperature range $15-600 \mathrm{~K}$ by Ragan et al. ${ }^{54}$ and was able to fit their data with very high confidence using line positions of $R_{1}$ obtained from Lorentzian fits to both $R_{1}$ and $R_{2}$ lines. We have confirmed that the temperature scale we used for the present $P$ - $T$ range gives a slightly higher pressure than the use of the cubic equation (at most $2.3 \%$, but typically about $1 \%$ ). However, this is approximately the same as the uncertainty in pressure associated with possible temperature errors discussed above.

Ruby fluorescence and Brillouin measurements were performed simultaneously using the same excitation; the ruby fluorescence shift was measured twice during acquisition of a Brillouin spectrum to confirm that the pressure did not vary during the measurement of the spectrum. It should be noted that the laser beam was focused on the sample at a position far from the ruby sphere to avoid local heating through absorption of the laser light by the ruby, which could cause a considerable error in determining temperature as well as pressure.

We typically began each run by setting the diamond cell at the desired pressure at room temperature, and then raising the temperature up to $530 \mathrm{~K}$. After slowly cooling the cell to room temperature, we set it to another pressure, and then followed another temperature run. It should be noted that pressure unintentionally varied as temperature was raised, particularly at melting points. We performed five sets of experiments; (1) initially at $4.6 \mathrm{GPa}$, and varied between 4.6 and $6.3 \mathrm{GPa}$; (2) at $6.7 \mathrm{GPa}, 6.7-7.3 \mathrm{GPa}$; (3) at $8.0 \mathrm{GPa}$, 7.5-8.3 GPa; (4) at $10.3 \mathrm{GPa}, 9.9-10.9 \mathrm{GPa}$; and (5) at 12.7 $\mathrm{GPa}, 12.0-13.8 \mathrm{GPa}$. At high pressures and temperatures, the rhenium gasket tended to degrade as a result of reaction with hydrogen. ${ }^{58}$ However, we confirmed that rhenium did not dissolve in fluid hydrogen causing possible shifts in the Brillouin signal from the sample.

Brillouin spectra were measured with a six-pass tandem Fabry-Perot interferometer. The $514.5 \mathrm{~nm}$ line from an $\mathrm{Ar}^{+}$ laser was used as the excitation source. Details for the highpressure Brillouin experiments can be found in Ref. 59. Brillouin data were collected using a $90^{\circ}$ scattering geometry (see Fig. 1), probing the sound wave direction parallel to the diamond culets. For the $90^{\circ}$ scattering geometry in an optically isotropic material, the compressional (longitudinal) sound velocity $U$ can be obtained by $U=\nu_{90} \lambda_{0} / \sqrt{2}$, where $\nu_{90}$ is the Brillouin frequency shift, and $\lambda_{0}$ is the wavelength of the incident light. In this geometry, $U$ is independent of the refractive index of the medium. There is also a laser beam reflected from the output diamond anvil that serves as incident light, giving a back-scattering signal $\left(180^{\circ}\right.$ scattering geometry) as shown in Fig. 1(b). In the $180^{\circ}$ scattering, the sound wave direction is not parallel to the diamond culets. The Brillouin frequency shift $\nu_{180}$ is related to $U$ by $U$ $=\nu_{180} \lambda_{0} /(2 n)$, where $n$ is the refractive index of the medium assumed to be optically isotropic. Both $\nu_{90}$ and $\nu_{180}$ can be detected simultaneously.

\section{RESULTS AND DISCUSSION}

\section{A. Brillouin scattering spectra}

Figure 2 shows a representative temperature dependence of Brillouin spectra at $P \approx 8.0 \mathrm{GPa}$. In the experiment, the 


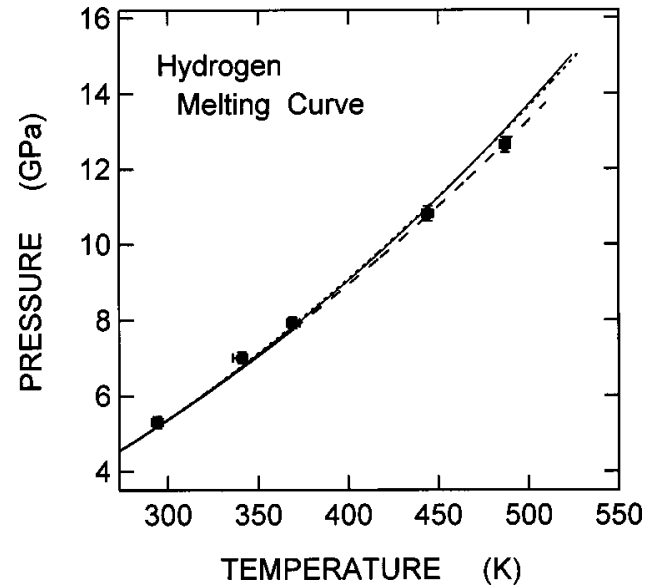

FIG. 3. Melting curve of hydrogen. Solid circles, present Brillouin scattering data; thick solid line, modified Simon-Glatzel equation fit from Diatschenko et al. (Ref. 43); dashed line, extrapolation of the modified Simon-Glatzel equation; thin solid line and dotted line, Kechin and SimonGlatzel equations, respectively, by Datchi et al. (Ref. 44). The horizontal error bar is due to possible temperature misreading estimated by the temperature difference between two thermocouples at different positions, plus the temperature variation during Brillouin measurements. The vertical error bar is drawn by the pressure uncertainty caused by the temperature error in the ruby pressure and temperature scale.

pressure changed as temperature was raised. The longitudinal acoustic (compressional) mode of hydrogen, the strongest peak in the figure, shifted abruptly to a lower frequency when temperature was raised from 364 to $376 \mathrm{~K}$, indicating the melting of solid hydrogen. It should be noticed that the fluid mode started to be observed at $7.9 \mathrm{GPa}, 364 \mathrm{~K}$. The temperature variation during the Brillouin measurement was about $2 \mathrm{~K}$. Because of two phase coexistence under the isoschoric conditions of the experiments, we infer that melting takes place at $364 \pm 1.0 \mathrm{~K}$ at $7.9 \mathrm{GPa}$. After the melting was completed, the pressure jumped to $8.3 \mathrm{GPa}$. The shear wave peak for the solid was not detected. Weak but appreciable back-scattering signals for the fluid were observed, as indicated in the figure.

\section{B. Melting curve}

The melting point $\left(T_{\mathrm{m}}, P_{\mathrm{m}}\right)$ defined as the temperature and the pressure at which the Brillouin peak for fluid hydrogen appears is plotted on our $P-T$ range in Fig. 3 . The melting curve of hydrogen has been determined 20 to $373 \mathrm{~K}$ by Diatschenko et al. ${ }^{43}$ and was fit to a modified Simon-Glatzel equation: $P_{\mathrm{m}}=-0.05149+1.702 \times 10^{-4}\left(T_{\mathrm{m}}\right.$ $+9.689)^{1.8077}$. Recently, the melting curve was extended to $525 \mathrm{~K}$ and $15 \mathrm{GPa}$ by direct visualization of melting of samples and optical techniques. ${ }^{44}$ The results were fit to Simon-Glatzel and Kechin equations: $P_{\mathrm{m}}=1.63$ $\times 10^{-4} T_{\mathrm{m}}^{1.824}$ and $T_{\mathrm{m}}=14.025\left(1+P_{\mathrm{m}} / 0.0286\right)^{0.589} \exp (-4.6$ $\left.\times 10^{-3} P_{\mathrm{m}}\right)$. The previously proposed melting lines are also shown in the figure. Our melting data are in reasonably good agreement with the extrapolation by the modified SimonGlatzel equation from Ref. 43, and deviate slightly from the Simon-Glatzel and Kechin equations from Ref. 44. Extrapolation of the proposed Kechin equation predicts that the melting curve of hydrogen would exhibit a maximum $T_{\mathrm{m}}$ of 1100
TABLE I. Sound velocity data for fluid hydrogen obtained in the present work. The estimated experimental uncertainty in $U$ is about $1.5 \%$ at $293 \mathrm{~K}$ and $1.7 \%$ at high temperatures.

\begin{tabular}{|c|c|c|}
\hline $\begin{array}{c}T \\
(\mathrm{~K})\end{array}$ & $\begin{array}{c}P \\
(\mathrm{GPa})\end{array}$ & $\begin{array}{l}U \text {, Sound velocity } \\
(\mathrm{km} / \mathrm{s})\end{array}$ \\
\hline 293 & 1.19 & 5.16 \\
\hline 293 & 1.6 & 5.77 \\
\hline 293 & 2.13 & 6.33 \\
\hline 293 & 2.57 & 6.72 \\
\hline 293 & 2.6 & 6.73 \\
\hline 293 & 3.2 & 7.14 \\
\hline 293 & 3.39 & 7.33 \\
\hline 293 & 3.8 & 7.46 \\
\hline 293 & 4.5 & 7.90 \\
\hline 293 & 4.58 & 8.04 \\
\hline 293 & 4.9 & 8.25 \\
\hline 293 & 5.03 & 8.28 \\
\hline 366 & 7.92 & 9.29 \\
\hline 376 & 8.34 & 9.38 \\
\hline 390 & 8.28 & 9.35 \\
\hline 409 & 8.18 & 9.32 \\
\hline 430 & 8.02 & 9.28 \\
\hline 448 & 7.84 & 9.24 \\
\hline 468 & 7.67 & 9.19 \\
\hline 491 & 7.47 & 9.12 \\
\hline 343 & 7.11 & 8.98 \\
\hline 356 & 7.16 & 9.03 \\
\hline 371 & 7.15 & 9.04 \\
\hline 395 & 7.21 & 9.07 \\
\hline 412 & 7.26 & 9.08 \\
\hline 432 & 7.24 & 9.10 \\
\hline 453 & 7.27 & 9.10 \\
\hline 478 & 7.07 & 9.09 \\
\hline 497 & 6.99 & 9.07 \\
\hline 445 & 10.81 & 10.31 \\
\hline 449 & 10.72 & 10.3 \\
\hline 463 & 10.84 & 10.22 \\
\hline 486 & 10.1 & 10.16 \\
\hline 501 & 9.89 & 10.08 \\
\hline 489 & 12.64 & 10.72 \\
\hline 496 & 12.42 & 10.68 \\
\hline 510 & 12.0 & 10.6 \\
\hline 335 & 4.94 & 8.25 \\
\hline 367 & 5.5 & 8.49 \\
\hline 400 & 5.96 & 8.72 \\
\hline 440 & 6.24 & 8.88 \\
\hline 485 & 6.42 & 8.94 \\
\hline 526 & 6.32 & 8.92 \\
\hline
\end{tabular}

$\mathrm{K}$ at $P_{\mathrm{m}}=128 \mathrm{GPa},{ }^{44}$ which implies that the fluid becomes denser than the solid on melting above that pressure. Subsequent measurements from our laboratory to $75 \mathrm{GPa}^{60}$ are consistent with the data reported in Ref. 44 and indicate a possible melting maximum at even lower pressure and temperature using the Kechin formulation. However, other fits to the data do not predict a melting point maximum. ${ }^{60}$ Accurate determination of the melting line in the higher pressure range is required to test this prediction.

\section{Equation of state}

The sound velocity data for the fluid obtained from the Brillouin spectra are tabulated in Table I. We first compared our sound velocity data with the extrapolated values of sound velocity calculated using the Benedict-type EOS derived 
from the previously reported volume and ultrasonic velocity data at temperatures from 75 to $307 \mathrm{~K}$ and pressures from 0.2 to $2 \mathrm{GPa}^{20} \mathrm{We}$ found that the extrapolation from their EOS deviates significantly from our data at $293-526 \mathrm{~K}$ at high pressures. Note that the deviation starts around $1.5 \mathrm{GPa}$, while our data and those of Pratesi et al. ${ }^{22}$ below $1.5 \mathrm{GPa}$ at room temperature are in good agreement. This lower pressure EOS overestimates the sound velocity by $8.9 \%$ at 7.1 $\mathrm{GPa}$ and $343 \mathrm{~K}$, and $10.9 \%$ at $12.6 \mathrm{GPa}$ and $489 \mathrm{~K}$, compared with our data (i.e., the deviation increases with pressure). This implies that fluid hydrogen is much softer than is predicted by the $P-V-T$ EOS obtained below $2 \mathrm{GPa}$ and 307 $\mathrm{K}$. This is consistent with previous findings regarding the extrapolation of lower pressure EOS to higher pressure for the solid hydrogen. ${ }^{14,16}$ For example, effective intermolecular potentials derived from low-pressure properties of solid hydrogen at $4 \mathrm{~K}$ up to $2.6 \mathrm{GPa}$ could not explain the higherpressure properties and solid hydrogen is more compressible than is predicted by previously proposed intermolecular potentials.

Several types of EOS have been proposed for fluids, and have a variety of strengths and limitations with regard to the kinds of substances and range of conditions to which each may be applied. ${ }^{61}$ Some of them are in a form that is simpler for thermodynamic calculations (integrations). The Benedict equation $^{62}$ has been used successfully to describe the EOS of fluid hydrogen in $P \leqslant 2 \mathrm{GPa}, T \leqslant 307 \mathrm{~K}$, and offered a good description in thermodynamic properties. ${ }^{20}$ Hence, we determined a Benedict type of $P-V-T$ EOS,

$$
V(P, T)=\sum_{m=1}^{3} \sum_{n=-2}^{2} A_{n, m} T^{n / 2} P^{-m / 3},
$$

for fluid hydrogen for $P \leqslant 15 \mathrm{GPa}, T \leqslant 550 \mathrm{~K}$ by leastsquares fitting made simultaneously to the Brillouin sound velocity data and to the volume and ultrasonic velocity data available over the range $75<T<600 \mathrm{~K}$ and $0.07<P$ $<2 \mathrm{GPa}^{5,18-20,63,64}$ In this fitting procedure, the velocity $U(P, T)$ was calculated from the volume $V(P, T)$ given by Eq. (2) using the thermodynamic equation:

$$
\begin{aligned}
U(P, T)= & V(P, T)\left[-M\left\{\left(\frac{\partial V(P, T)}{\partial P}\right)_{T}\right.\right. \\
& \left.\left.+\frac{T\left[(\partial V(P, T) / \partial T)_{P}\right]^{2}}{C_{P}(P, T)}\right\}\right]^{-1 / 2},
\end{aligned}
$$

where $M$ is the molecular weight. Here, we calculated the heat capacity at constant pressure, $C_{P}(P, T)$, from

$$
C_{P}(P, T)=C_{P_{0}}(T)-T \int_{P_{0}}^{P}\left(\frac{\partial^{2} V(P, T)}{\partial T^{2}}\right)_{P} d P,
$$

where we used $C_{P_{0}}(T)=0.1084 T-7.4519 T^{0.5}+205.774$ $-1617.14 T^{-0.5}+4394.09 T^{-1}(\mathrm{~J} / \mathrm{mole} \mathrm{K})$, which was obtained by fitting to the heat capacity data in $80-800 \mathrm{~K}$ at $P_{0}=0.1 \mathrm{GPa} .{ }^{64}$

The experimental $V_{\text {exp }}(P, T)$ and $U_{\text {exp }}(P, T)$, were combined to give a best-fit Benedict-type EOS by minimizing the sum $S$ of 264 data points $(l=140, k=124)$ according to

$$
\begin{aligned}
S= & \sum_{i=1}^{l} w_{i}\left|1-\frac{U_{\exp }\left(P_{i}, T_{i}\right)}{U\left(P_{i}, T_{i}\right)}\right|^{2} \\
& +\sum_{j=1}^{k} w_{j}\left|1-\frac{V_{\exp }\left(P_{j}, T_{j}\right)}{V\left(P_{j}, T_{j}\right)}\right|^{2} .
\end{aligned}
$$

Here, $w$ is the weight that should be proportional to the inverse square of the standard deviation for each data point. We assumed that the standard deviations are proportional to the experimental uncertainty, $\sigma^{\prime}$, in each measurement. In our fitting, we used previously reported data for sound velocity at $0.26-1.93 \mathrm{GPa}$ and $88-295 \mathrm{~K}$ (53 data points) by Mills et al. ${ }^{20,63}$ at $1.0-5.3 \mathrm{GPa}$ at $293 \mathrm{~K}$ (17 points) by Pratesi et al., ${ }^{22}$ and at 0.07 and $0.1 \mathrm{GPa}$ at $60-500 \mathrm{~K}(27$ points) by Vargaftik et al. ${ }^{64}$ together with our data in this work at $1.2-13 \mathrm{GPa}$ and $293-526 \mathrm{~K}$ (43 data points). For volume data, we used 53 data points at $0.26-1.93 \mathrm{GPa}$ and $88-295 \mathrm{~K}$ by Mills et al. ${ }^{20,63} 32$ data points at $0.07-0.26$ GPa and $173-423 \mathrm{~K}$ by Michels et al. ${ }^{18} 25$ data points at 0.1-0.65 GPa and 298-423 K by Tsiklis et al. ${ }^{19} 13$ data points at $0.1 \mathrm{GPa}$ at $160-550 \mathrm{~K}$ by Vargaftik et al. ${ }^{64}$ and one data point at $5.36 \mathrm{GPa}$ at $300 \mathrm{~K}$ obtained using the Vinet EOS for solid hydrogen ${ }^{14}$ plus $\Delta V_{\mathrm{m}}$ at this melting point. ${ }^{5}$ $\Delta V_{\mathrm{m}}$ is the volume change at $T_{\mathrm{m}}$. The inclusion of the additional datum at $5.36 \mathrm{GPa}$ at $300 \mathrm{~K}$ for volume is preferable to fill in the high-pressure region, since the experimental volume data for the fluid are limited to below $2 \mathrm{GPa}$ on static compression. This volume datum is derived from accurate experimental density determination in the solid ${ }^{14}$ and from a functional form obtained from the fit to experimental and theoretical data for $\Delta V_{\mathrm{m}}$ as a function of volume of the solid on the melting line. ${ }^{5}$ The uncertainty in $\Delta V_{\mathrm{m}}$ at $5.36 \mathrm{GPa}$ at $300 \mathrm{~K}^{5}$ corresponds to about $0.5 \%$ of the volume of the solid at this melting point. Thus this data point provides a check on the validity of the EOS given by the fit.

It should be noted that the data points used for the fitting are not spread uniformly over the wide $P$ - $T$ range but tend to weight more heavily certain regions. Thus, to correct the nonuniformity of the data set in the $P$ - $T$ range, we also weighted each term of the sum of Eq. (5) by the relative coverage of each data point on the $P-T$ plane (i.e., the inverse of the relative density, $d$, of data points on a limited $P-T$ range). The weights, $w$, used for the least-squares fitting are tabulated in Table II together with $\sigma^{\prime}$ and $d$. The values of $\sigma^{\prime}$ were either taken from literature or assumed based on their experimental techniques. Use of this fitting routine in the wide pressure and temperature range would lead us to a poorer EOS at $\mathrm{P}<2 \mathrm{GPa}$ and $\mathrm{T}<300 \mathrm{~K}$ relative to that of Mills et al. ${ }^{20}$ However, our goal is not to revise the Mills et al. EOS but to extend the fluid hydrogen EOS to higher pressures and temperatures while at the same time giving a reasonably good form ( $\leqslant 1 \%$ average deviation in $V$ and $U$ ) even at low pressures and temperatures.

Only 9 of the 15 coefficients, $A_{n, m}$, in Eq. (2) were used and adjusted to give best-fits to the experimental data for proper convergence. The obtained best-fit parameters, $A_{n, m}$, are tabulated in Table III. The temperature dependence of volume along several isobars was calculated from Eq. (2) using the best-fit parameters, and is shown in Fig. 4. The 
TABLE II. Weights used in our weighted least-squares fits to derive the Benedict-type of $P-V-T$ EOS for fluid hydrogen. $N$ is the number of data points, $\sigma^{\prime}$ is the experimental uncertainty, $d$ is the relative density of data points on the $P$-T range, and $w$ is the weight. The weights are relative values that are proportional to $1 / \sigma^{\prime 2}$ and $1 / d$.

\begin{tabular}{|c|c|c|c|c|c|}
\hline & $P-T$ range & $N$ & $\sigma^{\prime}(\%)$ & $d$ & $w$ \\
\hline \multicolumn{6}{|l|}{ Sound velocity } \\
\hline \multirow{2}{*}{ This work } & $1.2-5.0 \mathrm{GPa}, 293 \mathrm{~K}$ & 12 & 1.5 & 1 & 1 \\
\hline & $4.6-13 \mathrm{GPa}, 300-530 \mathrm{~K}$ & 31 & 1.7 & 0.2 & 3.9 \\
\hline \multicolumn{5}{|l|}{ (Refs. 20 and 63) } & 5.2 \\
\hline $\begin{array}{l}\text { Pratesi et al. } \\
\quad \text { (Ref. 22) }\end{array}$ & $1.0-5.3 \mathrm{GPa}, 293 \mathrm{~K}$ & 17 & 1.5 & 1 & 1 \\
\hline \multirow{2}{*}{$\begin{array}{l}\text { Vargaftik et al. } \\
\quad \text { (Ref. 64) }\end{array}$} & $0.07-0.1 \mathrm{GPa}, 60-300 \mathrm{~K}$ & 19 & 0.5 & 4 & 2.2 \\
\hline & $0.07-0.1 \mathrm{GPa}, 350-500 \mathrm{~K}$ & 8 & 1.0 & 2 & 1.1 \\
\hline \multicolumn{6}{|l|}{ Volume } \\
\hline $\begin{array}{c}\text { Mills et al. } \\
\text { (Refs. } 20 \text { and } 63 \text { ) }\end{array}$ & $0.26-1.93 \mathrm{GPa}, 88-295 \mathrm{~K}$ & 53 & 0.4 & 2.7 & 5.2 \\
\hline $\begin{array}{l}\text { Michels et al. } \\
\quad \text { (Ref. 18) }\end{array}$ & $0.07-0.26 \mathrm{GPa}, 173-423 \mathrm{~K}$ & 32 & 0.1 & 15 & 15 \\
\hline $\begin{array}{l}\text { Tsiklis et al. } \\
\text { (Ref. 19) }\end{array}$ & $0.1-0.65 \mathrm{GPa}, 298-423 \mathrm{~K}$ & 25 & 0.3 & 6 & 4.2 \\
\hline \multirow{2}{*}{$\begin{array}{l}\text { Vargaftik et al. } \\
\quad \text { (Ref. 64) }\end{array}$} & $0.1 \mathrm{GPa}, 60-300 \mathrm{~K}$ & 8 & 0.5 & 2 & 4.5 \\
\hline & $0.1 \mathrm{GPa}, 350-550 \mathrm{~K}$ & 5 & 1.0 & 1 & 2.2 \\
\hline $\begin{array}{l}\text { Solid EOS }+\Delta V_{m} \\
\text { (Refs. } 5 \text { and } 14)\end{array}$ & $5.36 \mathrm{GPa}, 300 \mathrm{~K}$ & 1 & 0.5 & 0.2 & 45 \\
\hline
\end{tabular}

deviation of the experimental data from the best-fit EOS is shown in Figs. 5 and 6 for the sound velocity and the volume, respectively. Our EOS for fluid hydrogen is found to be within a total average deviation of $0.84 \%$ from the volume data and $1.02 \%$ from the sound velocity data in this $P-T$ range. Here, the total average deviation is defined as $(1 / l)$ $\times\left\{\Sigma_{i=1}^{l}\left|\left\{U_{\exp }\left(P_{i}, T_{i}\right)-U\left(P_{i}, T_{i}\right)\right\} / U\left(P_{i}, T_{i}\right)\right|\right\}$ for the sound velocity data, likewise for the volume data. Our EOS is fairly satisfactory, even though we covered a wide $T$ and $P$ range for the fitting, including many data points at low pressures below $1 \mathrm{GPa}$. The low pressure data at $0.1 \mathrm{GPa}$ from Vargaftik et al. ${ }^{64}$ exhibit large deviation from our EOS especially at high temperatures above $350 \mathrm{~K}$. The Vargaftik et al. data are also largely discrepant with the extrapolation of the EOS by Mills et al. ${ }^{20}$ toward high temperatures. We suggest that the high temperature data from Ref. 64 have appreciable errors due to experimental difficulties with the measurements at that time.

Figure 7 compares the EOS derived in the present study with results previously reported for fluid hydrogen at room temperature. The sound velocity calculated from the EOS obtained in this work fits the experimental data very well, while the extrapolation of the EOS by Mills et al. ${ }^{20}$ deviates

TABLE III. Values of the best-fit parameters in the Benedict-type of $P-V-T$ EOS given by Eq. (2) for fluid hydrogen. The units, $\mathrm{cm}^{3} / \mathrm{mole}$, GPa, and $\mathrm{K}$, are used for $V, P$, and $T$, respectively, in Eq. (2).

\begin{tabular}{cccc}
\hline \hline$n$ & $A_{n, 1}$ & $A_{n, 2}$ & $A_{n, 3}$ \\
\hline-2 & 0 & 0 & 75.3598 \\
-1 & 37.862 & 0 & -25.0872 \\
0 & 15.2894 & -8.28279 & 3.14718 \\
1 & 0 & 0 & 0 \\
2 & -0.00421328 & 0.0131564 & 0.00210469 \\
\hline \hline
\end{tabular}

significantly from the data at high pressures. The power low dependence, $U=2.25 P^{0.317}$, given by Brody et al. ${ }^{21}$ significantly underestimates the sound velocity. Both the extrapolation of the EOS by Mills et al. ${ }^{20}$ and the data from Pratesi et $a .^{22}$ give lower density than the EOS of the present study (Fig. 7 inset). They underestimate the density at the melting point, compared with the experimental value indicated by the solid square. On the other hand, the EOS given in Ref. 65 overestimates the density. Our EOS shows a better agreement with the experimental value for density at the melting point at room temperature. However, our EOS differs from that by Mills et al. by $1.6 \%$ in volume at room temperature and $2 \mathrm{GPa}$, which is more than the stated average deviation (about $0.7 \%$ ) of the latter EOS. Mills et al. ${ }^{20}$ state that at the

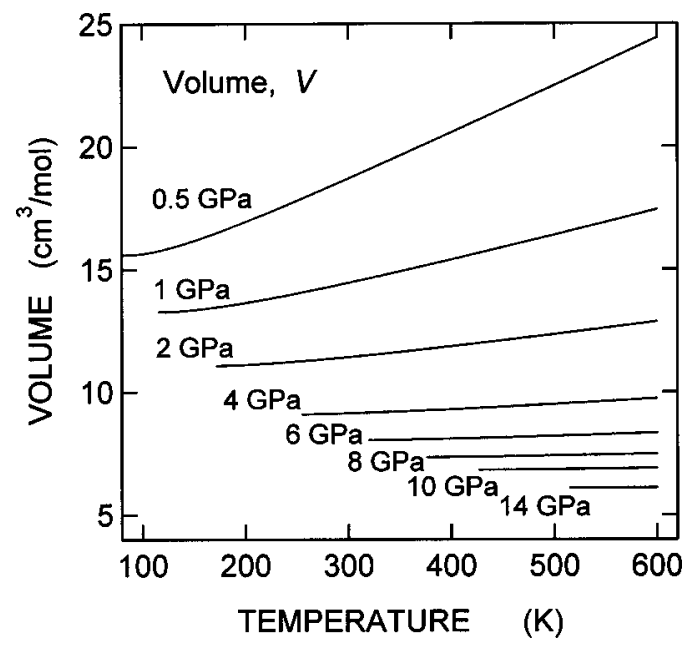

FIG. 4. Temperature dependence of volume along several isobars for fluid hydrogen calculated from the Benedict-type EOS with the best-fit parameters listed in Table III. 

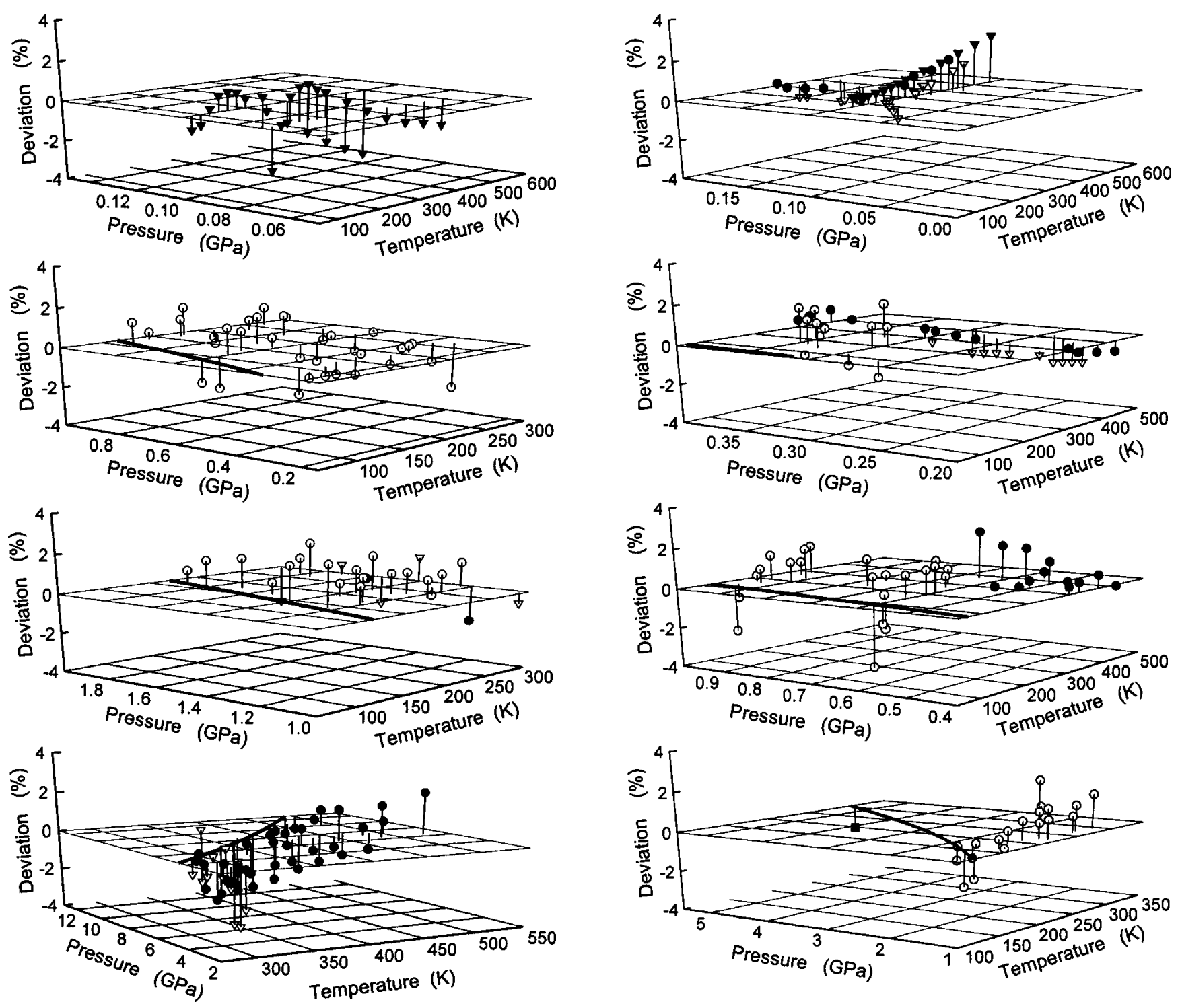

FIG. 5. Deviation of the experimentally derived sound velocity, $U_{\text {exp }}$, from the values calculated using the best-fit Benedict-type EOS, $U$. The deviation is defined as $\left(U_{\text {exp }}-U\right) / U$. - , present study; $\bigcirc$, Refs. 20 and 63; $\nabla$, Ref. 22; and $\boldsymbol{\nabla}$, Ref. 64. Vertical bars are drawn from each data point to the zerosurface to indicate the position $(T, P)$ and the distance in a perspective view. The thick solid line denotes the melting curve. The average relative deviation is $1.02 \%, 0.89 \%, 1.15 \%$, and $1.16 \%$ for $\bullet, \bigcirc, \nabla$, and $\boldsymbol{\nabla}$, respectively.

FIG. 6. Deviation of the volume data, $V_{\text {exp }}$, from the best-fit Benedict EOS, $V$. The deviation is defined as $\left(V_{\text {exp }}-V\right) / V ; \bigcirc$, Refs. 20 and 63; $\nabla$, Ref. 18; $\boldsymbol{0}$, Ref. $19 ; \boldsymbol{\nabla}$, Ref. 64 ; and $\mathbf{\square}$, the value estimated using the Vinet-type EOS for solid $n-\mathrm{H}_{2}$ at $300 \mathrm{~K}^{14}$ plus the volume change at the melting, $\Delta V_{m} .{ }^{5}$ Vertical bars are drawn from each data point to the zero-surface to indicate the position $(T, P)$ and the distance in a perspective view. The thick solid line denotes the melting curve. The average relative deviation is $1.02 \%, 0.60 \%, 0.67 \%, 1.05 \%$ and $1.19 \%$ for $\bigcirc, \nabla, \boldsymbol{\bullet}, \boldsymbol{\nabla}$, and respectively.

extrema of their dataset (temperatures $75 \mathrm{~K}$ or pressures of 2 GPa) where $P-T$ conditions were farthest from the point of normalization, an individual molar volume could stray by over $1 \%$. In addition, the data were not spread uniformly over their experimental $P-T$ range but tended to weight more heavily certain regions. Thus, the uncertainty in their EOS near the 2.0 GPa could be much larger than the average deviation of their EOS.

Although clearly beyond the expected range of extrapolation for our Benedict-type EOS, it is of interest to compare our EOS with $P-V-T$ data at extremely high pressures and temperatures where shock compression experiments and a variety of $a b$ initio calculations have been carried out. The volume at $3360 \mathrm{~K}$ for shock-compressed liquid hydrogen at $11.3 \mathrm{GPa}^{6}$ is larger than that extrapolated from our EOS by

$1.1 \%$, which is considered fairly good agreement. On the other hand, comparison with the results of density functional calculations at $1000 \mathrm{~K}^{28}$ shows that the volumes extrapolated from our EOS are larger than those calculated at $P=38$ and $230 \mathrm{GPa}$ by $14 \%$ and $17 \%$, respectively. In comparison to tight-binding molecular dynamic simulations ${ }^{66}$ the extrapolation of our EOS gives a volume within $4 \%$ at $19 \mathrm{GPa}$ and $4500 \mathrm{~K}$, and a smaller volume by $42 \%$ at $73 \mathrm{GPa}$ and 5000 K. Path-integral quantum Monte Carlo calculations ${ }^{67}$ and linear-mixing model calculations ${ }^{68}$ at $5000 \mathrm{~K}$ and $V$ $=6 \mathrm{~cm}^{3} / \mathrm{mol}$ have given the pressures of 79 and $28 \mathrm{GPa}$, respectively. In comparison to them, the volume extrapolated from our EOS is smaller by $65 \%$ than the former, and by $16 \%$ than the latter. Deviations of extrapolation of our EOS 


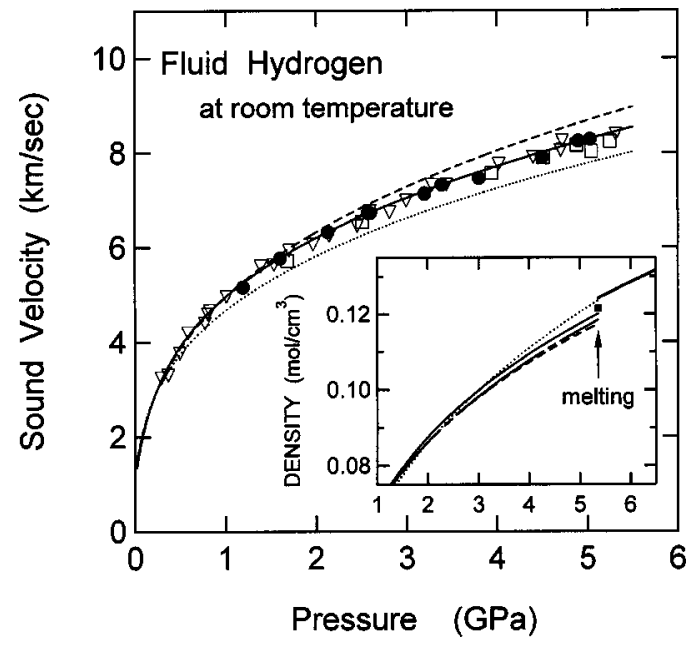

FIG. 7. Sound velocity as a function of pressure at room temperature for fluid hydrogen. - , present study; $\nabla$, Ref. 22; $\square$, Ref. 21; The solid line is calculated from the EOS of Eq. (2) using Eqs. (3) and (4) with the best-fit parameters listed in Table III. The dashed line is from the EOS by Mills et al. (Ref. 20) and its extrapolation. The dotted line is $U=2.25 P^{0.317}(P$ in kbar) given in Ref. 21 . The inset shows the pressure dependence of density, $\rho$, at room temperature. Solid line, EOS in the present study; dashed line, EOS by Mills et al. and its extrapolation; dotted line, $\rho=0.0577 P^{0.366}$ -0.0006 ( $\rho$ in $\mathrm{g} / \mathrm{cm}^{3}, P$ in kbar) given in Ref. 65; long dash-dotted line, $\rho=68.978 P^{0.321897}(\rho$ in mol/l, $P$ in GPa $)$ from Ref. 22 ; and $\mathbf{\square}$, the value obtained from the Vinet-type EOS for solid $n-\mathrm{H}_{2}$ at room temperature (Ref. 14) (shown by the thick solid line above $5.35 \mathrm{GPa}$ ) plus the volume change at the melting (Ref. 5).

from the above calculations become more significant at either higher temperatures or pressures where the dissociation to the high-density monatomic-metallic fluid is predicted to take place ${ }^{68}$ It should also be noted that our EOS exhibits unphysical irregularity when extrapolated to the high $P-T$ range above $30 \mathrm{GPa}$ and $5000 \mathrm{~K}$.

\section{Refractive index}

For an isotropic medium the ratio of $\nu_{180}$ to $\nu_{90}$, $\nu_{180} / \nu_{90}=\sqrt{2} n$, determines the value of the refractive index $n$. By combining the back-scattering and $90^{\circ}$ scattering signals, we obtained the refractive index for fluid hydrogen at 1.2 to $8.3 \mathrm{GPa}$ and 293 to $491 \mathrm{~K}$ (see Table IV). In Fig. 8(a), the refractive index is plotted as a function of density, together with room temperature data from Refs. 30 and 65. Here, the density was obtained from the EOS described in the previous section (Sec. III C). The refractive index, $n$, increases with density, $\rho$, following roughly the linear equation $n=1.0+1.61 \rho$, as shown in the figure. The refractive index is related to the polarizability, $\alpha$, by the Lorentz-Lorenz relation:

$$
\frac{\left(n^{2}-1\right)}{\left(n^{2}+2\right)}=\frac{4}{3} \pi\left(\frac{N_{A}}{V}\right) \alpha,
$$

where $N_{A}$ is the Avogadro's number. Using Eq. (6), we estimated $\alpha$ and plotted it as a function of $\rho$ in Fig. 8(b).

The refractive index for the solid has been determined by interference fringe measurements at $5 \mathrm{~K}$ up to $37 \mathrm{GPa} ;{ }^{69}$ the results were later corrected in Ref. 70. The index was also obtained by Brillouin scattering at room temperature up
TABLE IV. Refractive index $n$ of fluid hydrogen obtained in the present work. The estimated error in $n$ is about $2.0 \%$.

\begin{tabular}{ccc}
\hline \hline$T$ & $P$ & $n$ \\
$(\mathrm{~K})$ & $(\mathrm{GPa})$ & $\begin{array}{c}n \\
\text { Refractive index }\end{array}$ \\
\hline 293 & 1.19 & 1.256 \\
293 & 3.20 & 1.341 \\
293 & 3.40 & 1.347 \\
376 & 8.34 & 1.443 \\
390 & 8.28 & 1.445 \\
409 & 8.18 & 1.443 \\
430 & 8.02 & 1.438 \\
448 & 7.84 & 1.434 \\
468 & 7.67 & 1.429 \\
491 & 7.47 & 1.424 \\
343 & 7.11 & 1.423 \\
356 & 7.16 & 1.424 \\
371 & 7.15 & 1.429 \\
395 & 7.21 & 1.424 \\
412 & 7.26 & 1.423 \\
432 & 7.24 & 1.418 \\
453 & 7.27 & 1.422 \\
478 & 7.07 & 1.417 \\
\hline \hline
\end{tabular}

to $25 \mathrm{GPa}^{30,65}$ Shimizu et al. ${ }^{65}$ estimated $\alpha$ as a function of pressure and found that the polarizability of hydrogen was pressure insensitive, remaining at a constant value of 8.17 $\times 10^{-25} \mathrm{~cm}^{3}$ in the fluid phase, while it dropped discontinuously at the fluid-solid phase transition and then decreased continuously with further increase in pressure in the solid. A decrease in $\alpha$ with density was also observed by van Straaten et $\mathrm{al}^{70}$ for solid hydrogen above $1.5 \mathrm{GPa}$ at $5 \mathrm{~K}$ (above

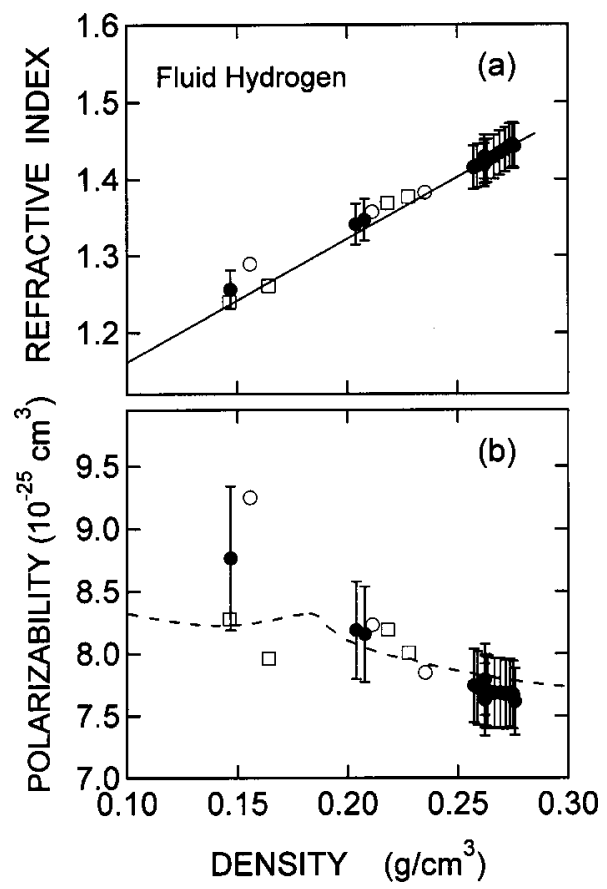

FIG. 8. (a) Refractive index and (b) polarizability as a function of density for fluid hydrogen. The polarizability was obtained from $n$ and $V(P, T)$ described in Sec. III C using the Lorentz-Lorenz relation of Eq. (6). Solid circles, present study at $1.2 \leqslant P \leqslant 8.3 \mathrm{GPa}$ and $293 \leqslant T \leqslant 491 \mathrm{~K}$; open circles and squares, room temperature data from Refs. 30 and 65 , respectively. The data for $n$ are fitted to $n=1.0+1.61 \rho$, as shown by the solid line in (a). The dashed line in (b) is from Ref. 70 for the solid at $5 \mathrm{~K}$. 


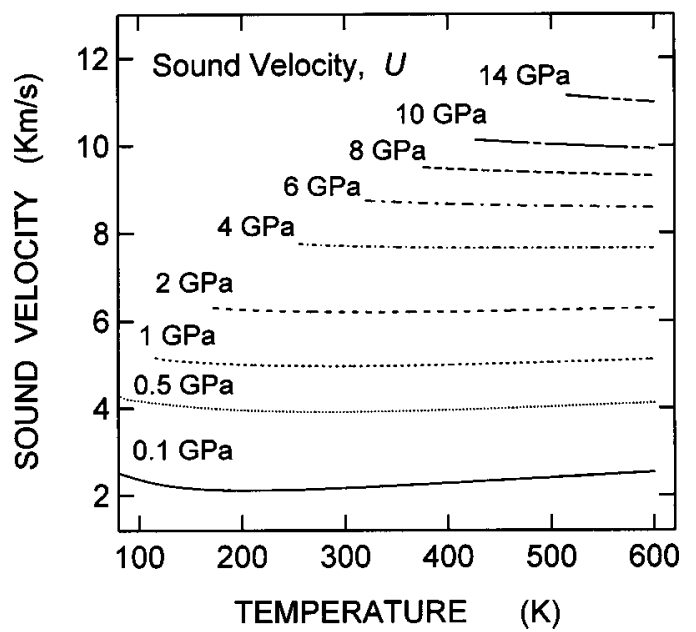

FIG. 9. Temperature dependence of sound velocity along several isobars for fluid hydrogen calculated from the EOS of Eq. (2) using Eqs. (3) and (4) with the best-fit parameters listed in Table III.

$0.184 \mathrm{~g} / \mathrm{cm}^{3}$ in density). Although effects of optical anisotropy have to be taken into account for $n$ in the solid phase, they were neglected in the previous work on solid hydrogen. This may lead to some errors on their estimation of $\alpha$ as a function of $\rho$. Here we extend the plot of $\alpha$ versus $\rho$ to a higher density region for fluid hydrogen using our data for $n$ at high $P-T$ and the more accurate EOS available now. As shown in Fig. 8(b), the polarizability of hydrogen decreases with density even in the fluid phase, which is in contrast with the previous findings. Our result for the fluid phase is reasonably in agreement with the revised result obtained for the solid by van Straaten et al. ${ }^{70}$ (shown by the dashed line in the figure), even though their data have considerably large uncertainties due to their determination of $n$ and neglect of optical anisotropy.

\section{E. Thermodynamic properties}

Using the EOS for fluid hydrogen, we calculated the pressure and temperature dependences of thermodynamic properties such as isobaric thermal expansion coefficient $\alpha_{P}=V^{-1}(\partial V / \partial T)_{P}$, isothermal and adiabatic compressibilities $\chi_{T}=-V^{-1}(\partial V / \partial P)_{T}$ and $\chi_{S}=V / M U^{2}$, heat capacities at constant pressure $C_{P}$ [from Eq. (4)] and at constant volume $C_{V}=C_{P} \chi_{S} / \chi_{T}$, and heat capacity ratio $\gamma=C_{P} / C_{V}$ $=\chi_{T} / \chi_{S}$. The velocity $U$ is plotted as a function of temperature along several isobars from 0.1 to $14 \mathrm{GPa}$ in Fig. 9. The curves are terminated at the freezing line calculated by the extrapolation of the modified Simon-Glatzel equation from Ref. 43 described in Sec. III B. Although the temperature coefficient of sound velocity in the isobars, $(\partial U / \partial T)_{P}$, is small, $U$ exhibits an intriguing temperature dependence. At low pressures below $6 \mathrm{GPa}, U$ decreases with an increase in temperature, and then begins to increase with temperature. This trend is consistent with the data in Ref. 64. The turnaround temperature $T_{\mathrm{t}}$ increases with an increase in pressure; i.e., $T_{\mathrm{t}}=210,285,300,335$, and 490 at $0.1,0.5,1,2$, and 4 $\mathrm{GPa}$, respectively. Above $6 \mathrm{GPa}$, the sound velocity decreases slightly and monotonically with an increase in temperature in the measured $P-T$ range. Similar plots of $\alpha_{P}, \chi_{T}$, $C_{P}$, and $C_{V}$ are presented along several isobars in Fig. 10. The heat capacity ratio $\gamma=C_{P} / C_{V}$, which is related to the dynamic structure factor, is a particularly important thermodynamic quantity, as $\gamma$ can be used generally to obtain the sound velocity along isotherms from the $P-V$ EOS. The values for $\gamma$ are plotted as a function of temperature along eight isobars from 0.5 to $14 \mathrm{GPa}$ in Fig. 11(a) and as a function of pressure along nine isotherms from 150 to $600 \mathrm{~K}$ in Fig. 11(b). Along the isobars, $\gamma$ is close to 1.0 at the melting points (where the curves terminate in the figure), and increases with temperature. Along the isotherms, the $\gamma$ curves follow a smooth trend, dropping with increasing pressures up
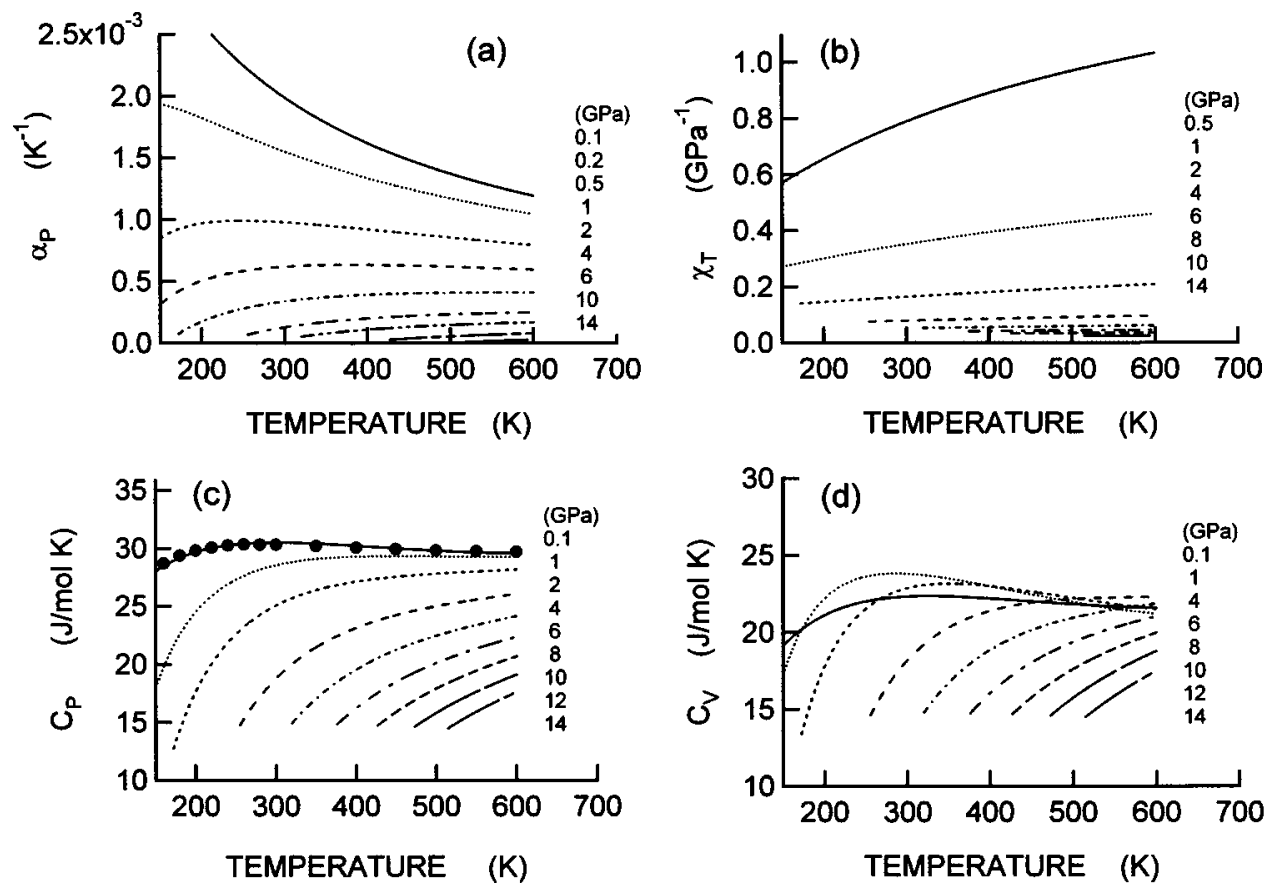

FIG. 10. Temperature dependences of thermodynamic properties along isobars for fluid hydrogen calculated from the best-fit EOS of Eq. (2). (a) Isobaric thermal expansion coefficient, $\alpha_{P}$; (b) isothermal compressibility, $\chi_{T}$; (c) heat capacity at constant pressure, $C_{P} ;$ (d) heat capacity at constant volume $C_{V}$. Solid circles in (c) denotes experimental data from Ref. 64. 

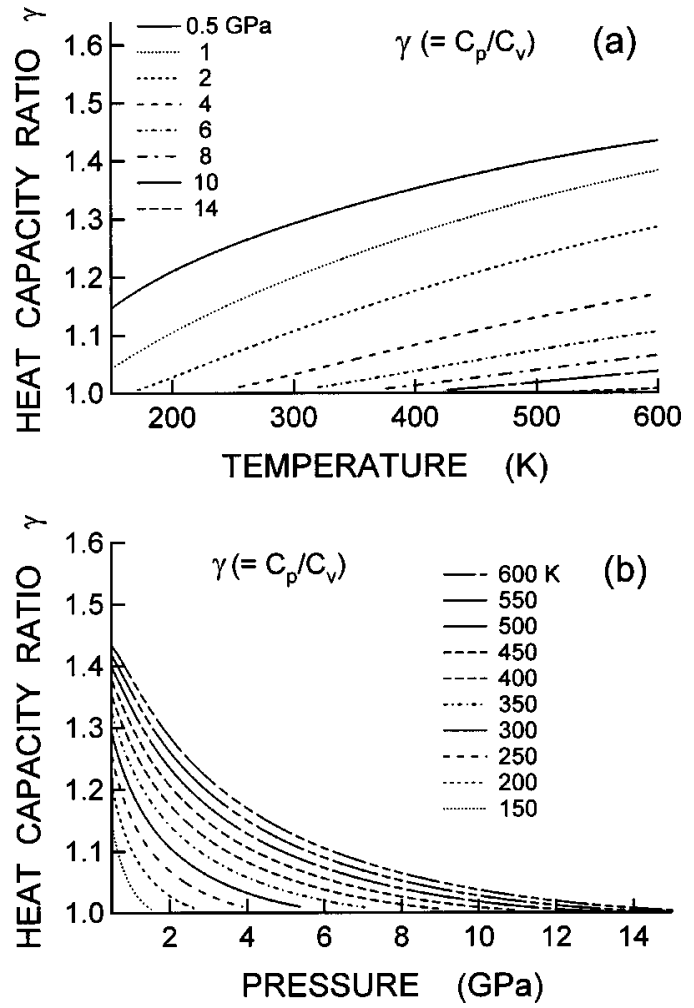

FIG. 11. (a) Heat capacity ratio, $\gamma=C_{P} / C_{V}$, as a function of temperature along isobars, and (b) $\gamma$ as a function of pressure along isotherms for fluid hydrogen.

to the freezing point before they reach 1.0. The extrapolation of $\gamma$ toward lower pressures gives good agreement with the values of hydrogen gas at atmospheric pressure; $\gamma=1.38$ at $300 \mathrm{~K}$ and $\gamma=1.40$ at $400 \mathrm{~K}^{71}$

\section{F. Intermolecular potential}

Accurate studies of the $P-V$ EOS of solid hydrogen at high pressures and room temperature (beginning at $5.4 \mathrm{GPa}$ ) have been performed with single-crystal diffraction. ${ }^{12-16}$ High-pressure $P-V$ EOS and elasticity data ${ }^{14,30}$ provided an important basis for evaluating effective pair potentials proposed for solid hydrogen. ${ }^{14,27}$ Effective pair potentials derived from low-pressure properties of the solid at $4 \mathrm{~K}$ up to $2.6 \mathrm{GPa}^{26}$ and from dynamic compression data for the fluid ${ }^{32}$ provided a poor fit to the properties of the solid (e.g., up to $\sim 30 \mathrm{GPa}) .{ }^{14,27}$ As discussed above, fluid hydrogen is also softer than predicted previously by the $P-V-T$ EOS for pressures below $2 \mathrm{GPa}$, suggesting that short range corrections to the intermolecular potential would be needed at high pressures and temperatures. Accordingly, it is useful to examine effective pair potentials appropriate for fluid hydrogen using our $P-V$ - $T$ EOS results up to $15 \mathrm{GPa}$ and $550 \mathrm{~K}$.

We examine three effective pair potentials for hydrogen previously proposed to fit experimental data for the material in both the solid and fluid states. In so doing, we also examine the broader question of whether a state-independent potential can fit the available data for both phases. An isotropic pair potential with a pair-wise (sphericalized) treatment of the Axilrod-Teller three-body term was developed by Sil- vera and Goldman ${ }^{26}$ to fit experimental data for low-density solid hydrogen at $4 \mathrm{~K}$ up to $2.6 \mathrm{GPa}$. The Silvera-Goldman (SG) potential is given by the form

$$
\begin{aligned}
V_{\mathrm{SG}}(r)= & \exp \left(\alpha-\beta r-\gamma r^{2}\right)-\left(\frac{C_{6}}{r^{6}}+\frac{C_{8}}{r^{8}}+\frac{C_{10}}{r^{10}}\right) f(r) \\
& +\frac{C_{9}}{r^{9}} f(r),
\end{aligned}
$$

where $f(r)$ is the damping function:

$$
\begin{aligned}
f(r) & =\exp \left[-\left(\frac{1.28 r_{m}}{r}-1\right)^{2}\right], \quad r<1.28 r_{m}, \\
& =1.0, \quad r \geqslant 1.28 r_{m} .
\end{aligned}
$$

Here $r$ is the intermolecular spacing, and $r_{m}$ is the potential well minimum $\left(r_{m}=6.444 \mathrm{bohr}\right)$. The parameters in Eq. (7) are given as follows: $\alpha=1.713, \quad \beta=1.5671, \quad \gamma=9.93$ $\times 10^{-3}, C_{6}=12.14, C_{8}=2.152 \times 10^{2}, C_{9}=1.431 \times 10^{2}$, and $C_{10}=4.8139 \times 10^{3}$ atomic units. The $C_{9}$ term contains the sphericalized Axilrod-Teller contribution. The SG potential successfully reproduced fluid isotherms of hydrogen from 75 to $300 \mathrm{~K}$ up to $2.0 \mathrm{GPa}^{20}$ and the melting curve to 5.7 $\mathrm{GPa}^{42,72}$ but could not predict the Hugoniot curves by shock experiments. ${ }^{24,32}$ Hence Ross et al. ${ }^{24,25}$ proposed a modified effective potential (Ross-Ree-Young, RRY) by softening the SG potential at short range as follows:

$$
\begin{aligned}
V_{\mathrm{RRY}}(r)= & V_{\mathrm{SG}}(r), \quad r \geqslant r_{C}, \\
= & A \exp \left[-B\left(r-r_{C}\right)-C\left(r-r_{C}\right)^{2}-D\left(r-r_{C}\right)^{3}\right. \\
& \left.-E\left(r-r_{C}\right)^{3}\left(r-r_{1}\right)\right], \quad r<r_{C},
\end{aligned}
$$

where $\quad A=3.98823 \times 10^{-14} \mathrm{erg}, \quad B=4.76940 \AA^{-1}, \quad C$ $=2.25457 \AA^{-2}, D=0.955189 \AA^{-3}, E=0.248158 \AA^{-4}, r_{1}$ $=1.2 \AA$, and $r_{C}=2.55 \AA$. Calculation of the EOS for the solid with this potential, however, significantly overestimated the pressure over the range measured in subsequent single-crystal $\mathrm{x}$-ray and neutron diffraction experiments to $30 \mathrm{GPa}^{13-15}$ This indicated that the effective potential was either strongly state- or temperature-dependent (rendering untenable the notion and utility of an effective potential approach). Alternatively, further softening of the potential was required to fit both the static and dynamic compression data. A further correction to the SG potential that fit to the x-ray diffraction data was examined together with roomtemperature sound velocity data for the solid. ${ }^{30}$ This potential (called Hemley-Silvera-Goldman, HSG) was also shown ${ }^{27}$ to give an excellent fit to both single- and double-shock Hugoniot data. ${ }^{32}$ The HSG represents the effective isotropic pair-wise interactions by including many-body terms implicitly in the form

$$
V_{\mathrm{HSG}}(r)=V_{\mathrm{SG}}(r)+V_{\mathrm{SR}}(r),
$$

where $V_{\mathrm{SR}}(r)$ is the potential for an $a d$ hoc short-range term given by

$$
\begin{aligned}
V_{\mathrm{SR}}(r) & =a_{1}\left(r-r_{c}\right)^{3}+a_{2}\left(r-r_{c}\right)^{6}, \quad r \leqslant r_{c}, \\
& =0, \quad r>r_{c} .
\end{aligned}
$$



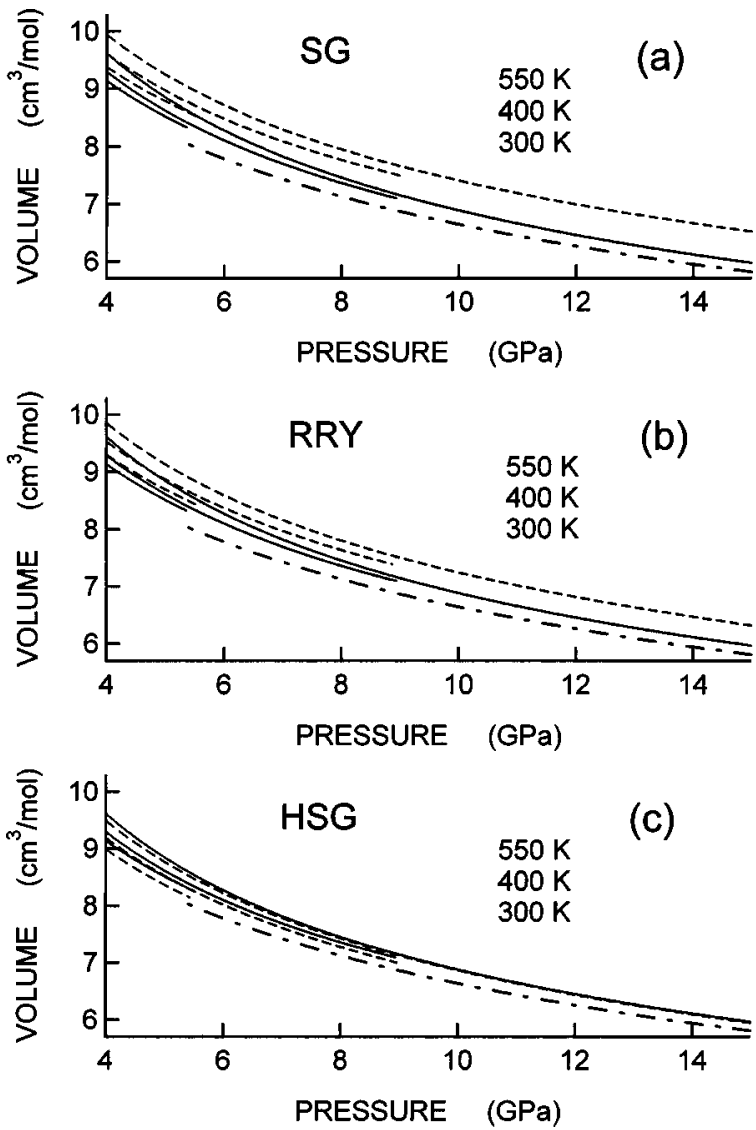

FIG. 12. Comparison of the Benedict-type EOS derived for fluid hydrogen in the present study with those calculated by fluid perturbation theory using the (a) SG, (b) RRY, and (c) HSG potentials along the isotherms at 300, 400, and $550 \mathrm{~K}$. Solid lines are for the experimental EOS, and dashed lines for the calculations. The dash-dot line denotes the Vinet-type EOS for solid hydrogen at room temperature given in Ref. 14.

The parameters used are $a_{1}=4.213 \times 10^{-4}$ hartree $/ \mathrm{bohr}^{3}$,

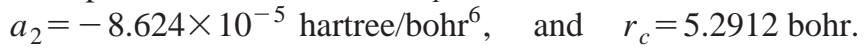
The three effective pair potentials described above were compared to experimental data obtained by both static compression of the solid and shock-wave data for the fluid. ${ }^{27}$

We now compare our experimentally derived EOS for fluid hydrogen in the $P$ - $T$ range of the present studies with the predictions of calculations using the three above potentials using fluid perturbation theory ${ }^{73}$ (see also Ref. 27). In Fig. 12, the EOS calculated from the fluid perturbation theory using the SG, RRY, and HSG potentials are compared with the Benedict-type EOS derived experimentally for fluid hydrogen along isotherms at 300,400 , and $550 \mathrm{~K}$ from 4 to $15 \mathrm{GPa}$. As is seen clearly, the SG and RRY potentials give significant deviations from the experimental EOS as pressure increases along these isotherms. On the other hand, the experimental EOS is reproduced reasonably well using the HSG potential above $6 \mathrm{GPa}$.

Figure 13 shows the volume calculated using the three potentials together with the experimental EOS as a function of temperature from the melting line to $600 \mathrm{~K}$ along isobars at $1,3,6$, and $13 \mathrm{GPa}$. As may be expected, the SG and RRY potentials give fairly good fits at low $P-T$ (for example, below $300 \mathrm{~K}$ at $1 \mathrm{GPa}$ ), but there are differences with the experimental EOS at the high $P-T$ range. Above $6 \mathrm{GPa}$, the

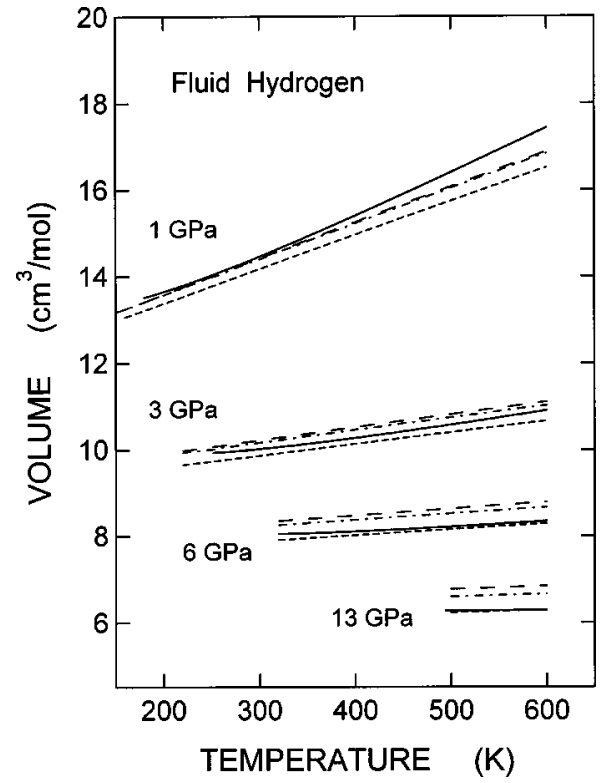

FIG. 13. Temperature dependence of volume along isobars calculated using the SG, RRY, and HSG potentials. Long-dash line, SG; dash-dot line, RRY; short-dash line, HSG. The solid lines are from the Benedict-type EOS derived for fluid hydrogen in the present study.

HSG potential gives a good fit over a wide temperature range. Although further improvement could be sought, the comparison indicates that the HSG potential is a useful description of the effective intermolecular interactions for the fluid in the given $P-T$ range.

Since the HSG potential was shown to fit high-pressure $\mathrm{x}$-ray data at room temperature for the solid phase (i.e., with quasiharmonic lattice dynamics calculations ${ }^{14}$ ), the good agreement for the fluid phase above $6 \mathrm{GPa}$ up to $600 \mathrm{~K}$ as well indicates that at least in this $P-T$ range an effective, state-independent pair potential can be used to describe the EOS and therefore other thermodynamic properties. However, we emphasize that none of the potentials can reproduce satisfactorily our experimental EOS in the high temperature range at low pressures (i.e., for low-density fluid). The calculation using the potentials underestimates the volume by more than $1.5 \%$ at $450 \mathrm{~K}$ at $1 \mathrm{GPa}$ compared with the experimentally derived EOS. Our EOS is within $0.6 \%$ volume deviation from Michels' data at $0.07-0.3 \mathrm{GPa}$ and $173-423$ $\mathrm{K}$ and within $0.7 \%$ deviation from Tsiklis' data at $0.1-0.65$ GPa and 298-423 K. Thus, the discrepancy between experimental and calculated EOS at high temperatures at low pressures is not within the error, and provides another constraint on determination of the intermolecular potential. Differences at this level point to the possible limitations in the use of temperature- and state-independent potentials that neglect differences in rotational, vibrational, and even electronic properties in different regions of pressure and temperatures. Indeed, at high $P-T$ conditions reached in shock experiments, there is a need to include partial dissociation and changes in electronic properties. Within the $P-T$ range of the present study, however, the agreement between the measurements and the calculations (i.e., with the HSG potential) indicate that major changes in the bonding properties of hydrogen do not occur. 


\section{CONCLUSION}

We have investigated the temperature dependences of the sound velocity of fluid hydrogen $\left(n-\mathrm{H}_{2}\right)$ by Brillouin measurements at high pressures (1 to $13 \mathrm{GPa}$ ) and temperatures (293-526 K). From sound velocity data together with existing volume and ultrasonic velocity data at low pressures and temperatures, we determined a Benedict-type $P-V-T$ EOS valid up to $15 \mathrm{GPa}$ and $550 \mathrm{~K}$ for fluid hydrogen with an average deviation of $1.0 \%$ from the existing experimental data. Using the EOS, we calculated the pressure and temperature dependences of thermodynamic properties, including thermal expansion coefficient, isothermal and adiabatic compressibilities, heat capacities at constant volume $C_{V}$ and at constant pressure $C_{p}$, and heat capacity ratio $\gamma$ $=C_{p} / C_{V}$. Comparison of the EOS with the volume calculated using different effective pair potentials using fluid perturbation theory leads us to conclude that the HSG potential is a useful description of intermolecular interactions for fluid hydrogen in this $P-T$ range. The discrepancies provide another constraint on determination of the effective intermolecular potential, although its use over a wide $P-T$ range would likely require the incorporation of additional channels such as dissociation.

\section{ACKNOWLEDGMENTS}

The authors would like to thank Y. Fei for assistance with the high-temperature diamond-cell technique. We are also grateful to T. S. Duffy and W. L. Vos for use of the fluid perturbation theory code. K.M. acknowledges financial support from the Venture Business Laboratory at the University of Tsukuba and the Ministry of Education, Culture, Sports, Science and Technology in Japan. This work was also supported by the NSF and NASA.

${ }^{1}$ H. K. Mao and R. J. Hemley, Rev. Mod. Phys. 66, 671 (1994).

${ }^{2}$ R. J. Hemley and H. K. Mao, J. Low Temp. Phys. 122, 331 (2001).

${ }^{3}$ J. Kohanoff, J. Low Temp. Phys. 122, 297 (2001), and references therein.

${ }^{4}$ N. W. Ashcroft, in Proceedings of the International School of Physics

"Enrico Fermi," Course CXLVII, High Pressure Phenomena, edited by R.

J. Hemley, G. L. Chiarotti, M. Bernasconi, and L. Ulivi (IOS, Amsterdam, 2002), p. 151.

${ }^{5}$ H. Hemmes, A. Driessen, and R. Griessen, J. Phys. C 19, 3571 (1986).

${ }^{6}$ N. C. Holmes, M. Ross, and W. J. Nellis, Phys. Rev. B 52, 15835 (1995).

${ }^{7}$ S. T. Weir, A. C. Mitchell, and W. J. Nellis, Phys. Rev. Lett. 76, 1860 (1996); W. J. Nellis, S. T. Weir, and A. C. Mitchell, Phys. Rev. B 59, 3434 (1999).

${ }^{8}$ G. W. Collins, L. B. Da Silva, P. Celliers et al., Science (Washington, DC, U.S.) 281, 1178 (1998).

${ }^{9}$ Several EOS models for shock-compressed deuterium are discussed in High Pressure Research 16, 281-400 (2000), a special issue entitled $\mathrm{Hy}$ drogen at High Pressure, edited by D. A. Young and R. Cauble.

${ }^{10}$ P. M. Celliers, G. W. Collins, L. B. Da Silva, D. M. Gold, R. Cauble, R. J. Wallace, M. E. Foord, and B. A. Hammel, Phys. Rev. Lett. 84, 5564 (2000); G. W. Collins, P. M. Celliers, L. B. Da Silva, R. Cauble, D. M. Gold, M. E. Foord, N. C. Holmes, B. A. Hammel, and R. J. Wallace, ibid. 87, 165504 (2001)

${ }^{11}$ D. J. Stevenson, in Proceedings of the International School of Physics "Enrico Fermi," Course CXLVII, High Pressure Phenomena, edited by R. J. Hemley, G. L. Chiarotti, M. Bernasconi, and L. Ulivi (IOS, Amsterdam, 2002), p. 587.

${ }^{12}$ H. K. Mao, A. P. Jephcoat, R. J. Hemley, L. W. Finger, C. S. Zha, R. M. Hazen, and D. E. Cox, Science (Washington, D.C., U.S.) 239, 1131 (1988).

${ }^{13}$ V. P. Glazkov, S. P. Besedin, I. N. Goncharenko, A. V. Irodova, I. N.
Makarenko, V. A. Somenkov, S. M. Stishov, and S. S. Shilsteyn, JETP Lett. 47, 661 (1988).

${ }^{14}$ R. J. Hemley, H. K. Mao, L. W. Finger, A. P. Jephcoat, R. M. Hazen, and C. S. Zha, Phys. Rev. B 42, 6458 (1990).

${ }^{15}$ J. Hu, H. K. Mao, J. F. Shu, and R. J. Hemley, in High Pressure Science and Technology, edited by S. C. Schmidt, J. W. Shaner, G. A. Samara, and M. Ross (AIP, New York, 1994), p. 441.

${ }^{16}$ P. Loubeyre, R. LeToullec, D. Hausermann, M. Hanfland, R. J. Hemley, H. K. Mao, and L. W. Finger, Nature (London) 383, 702 (1996)

${ }^{17}$ H. Kawamura, Y. Akahama, S. Umemoto, K. Takemura, Y. Ohishi, and O. Shimomura, Solid State Commun. 119, 29 (2001).

${ }^{18}$ A. Michels, W. De Graaff, T. Wassenaar, J. M. H. Levelt, and P. Louwerse, Physica (Amsterdam) 25, 25 (1959).

${ }^{19}$ D. S. Tsiklis, V. Ya Maslennikova, S. D. Gavrilov, A. N. Egorov, and G. V. Timofeeva, Dokl. Akad. Nauk SSSR 220, 1384 (1975).

${ }^{20}$ R. L. Mills, D. H. Liebenberg, L. C. Bronson, and L. C. Schmidt, J. Chem. Phys. 66, 3076 (1977).

${ }^{21}$ E. M. Brody, H. Shimizu, H. K. Mao, P. M. Bell, and W. A. Bassett, J. Appl. Phys. 52, 3583 (1981).

${ }^{22}$ G. Pratesi, L. Ulivi, F. Barocchi, P. Loubeyre, and R. LeToullec, J. Phys.: Condens. Matter 9, 10059 (1997).

${ }^{23}$ H. Shimizu, Mater. Res. Soc. Symp. Proc. (Materials Research Society, Pittsburgh, 1984), Vol. 22, p. 57.

${ }^{24}$ M. Ross, F. H. Ree, and D. A. Young, J. Chem. Phys. 79, 1487 (1983).

${ }^{25}$ W. J. Nellis, M. Ross, A. C. Mitchell, M. van Thiel, D. A. Young, F. H. Ree, and R. J. Trainor, Phys. Rev. A 27, 608 (1983).

${ }^{26}$ I. F. Silvera and V. V. Goldman, J. Chem. Phys. 69, 4209 (1978).

${ }^{27}$ T. S. Duffy, W. L. Vos, C. S. Zha, R. J. Hemley, and H. K. Mao, Science (Washington, D.C., U.S.) 263, 1590 (1994).

${ }^{28}$ A. Alavi, M. Parrinello, and D. Frenkel, Science (Washington, D.C., U.S.) 269, 1252 (1995).

${ }^{29}$ W. J. Nellis, M. Ross, and N. C. Holmes, Science (Washington, D.C., U.S.) 269, 1249 (1995).

${ }^{30}$ C. S. Zha, T. S. Duffy, H. K. Mao, and R. J. Hemley, Phys. Rev. B 48, 9246 (1993)

${ }^{31}$ J. Kohanoff and J. P. Hansen, Phys. Rev. E 54, 768 (1996).

${ }^{32}$ W. J. Nellis, A. C. Mitchell, M. van Thiel, G. J. Devine, R. J. Trainor, and N. Brown, J. Chem. Phys. 79, 1480 (1983).

${ }^{33}$ L. B. Da Silva, P. Celliers, G. W. Collins et al., Phys. Rev. Lett. 78, 483 (1997).

${ }^{34}$ A. N. Mostovych, Y. Chan, T. Lehecha, A. Schmitt, and J. D. Sethian, Phys. Rev. Lett. 85, 3870 (2000).

${ }^{35}$ M. D. Knudson, D. L. Hanson, J. E. Bailey, C. A. Hall, J. R. Asay, and W. W. Anderson, Phys. Rev. Lett. 87, 225501 (2001).

${ }^{36}$ W. J. Nellis, Phys. Rev. Lett. 89, 165502 (2002).

${ }^{37}$ T. J. Lenosky, S. R. Bickham, J. D. Kress, and L. A. Collins, Phys. Rev. B 61, 1 (2000)

${ }^{38}$ G. Galli, R. Q. Hood, A. U. Hazi, and F. Gygi, Phys. Rev. B 61, 909 (2000)

${ }^{39}$ M. Ross and L. H. Yang, Phys. Rev. B 64, 174102 (2001).

${ }^{40}$ B. Militzer, D. M. Ceperley, J. D. Kress, J. D. Johnson, L. A. Collins, and S. Mazevet, Phys. Rev. Lett. 87, 275502 (2001).

${ }^{41}$ P. Loubeyre, P. M. Celliers, D. G. Hicks et al. (private communication).

${ }^{42}$ V. Diatschenko and C. W. Chu, Science (Washington, D.C., U.S.) 212, 1393 (1981)

${ }^{43}$ V. Diatschenko, C. W. Chu, D. H. Liebenberg, D. A. Young, M. Ross, and R. L. Mills, Phys. Rev. B 32, 381 (1985).

${ }^{44}$ F. Datchi, P. Loubeyre, and R. LeToullec, Phys. Rev. B 61, 6535 (2000).

${ }^{45}$ K. Matsuishi, E. Gregoryanz, H. K. Mao, and R. J. Hemley, J. Phys.: Condens. Matter 14, 10631 (2002).

${ }^{46}$ Y. Fei, in Mineral Spectroscopy: A Tribute to Roger G. Burns, edited by M. D. Dyar, C. McCammon, and M. W. Schafer (Geochemical Society, Houston, 1996), p. 243.

${ }^{47}$ D. E. McCumber and M. D. Sturge, J. Appl. Phys. 34, 1682 (1963).

${ }^{48}$ J. Yen and M. Nicol, J. Appl. Phys. 72, 5535 (1992).

${ }^{49}$ D. M. Adams, R. Appleby, and S. K. Sharma, J. Phys. E 9, 1140 (1976).

${ }^{50}$ S. Buchsbaum, R. L. Mills, and D. Schiferl, J. Phys. Chem. 88, 2522 (1984).

${ }^{51}$ J. D. Barnett, S. Block, and G. J. Piermarini, Rev. Sci. Instrum. 44, 1 (1973).

${ }^{52}$ S. Yamaoka, O. Shimomura, and O. Fukunaga, Proc. Jpn. Acad., Ser. B: Phys. Biol. Sci. 56, 103 (1980). 
${ }^{53}$ R. G. Munro, G. J. Piermarini, S. Block, and W. B. Holzapfel, J. Appl. Phys. 57, 165 (1985).

${ }^{54}$ D. D. Ragan, R. Gustavsen, and D. Schiferl, J. Appl. Phys. 72, 5539 (1992).

${ }^{55}$ R. LeToullec and P. Loubeyre, J. Appl. Phys. 81, 3333 (1997).

${ }^{56}$ H. K. Mao, J. Xu, and P. M. Bell, J. Geophys. Res. 91, 4673 (1986).

${ }^{57}$ R. A. Noack and W. B. Holzapfel, in High Pressure Science and Technology, edited by K. D. Timmerhaus and M. S. Barber (Plenum, New York, 1979), Vol. 1, p. 748.

${ }^{58}$ J. V. Badding, R. J. Hemley, and H. K. Mao, Science (Washington, D.C., U.S.) 253, 421 (1991).

${ }^{59}$ C. S. Zha, T. S. Duffy, R. T. Downs, H. K. Mao, and R. J. Hemley, J. Geophys. Res. 101, 17535 (1996).

${ }^{60}$ E. Gregoryanz, A. F. Goncharov, K. Matsuishi, H. K. Mao, and R. J. Hemley, Phys. Rev. Lett. (to be published).

${ }^{61}$ For example, see M. J. Assael, J. P. M. Trusler, and T. F. Tsolakis, in Thermodynamic Properties of Fluids (Imperial College, London, 1996), Chap. 6.

${ }^{62}$ M. Benedict, J. Am. Chem. Soc. 59, 2233 (1937).
${ }^{63}$ D. H. Liebenberg, R. L. Mills, and L. C. Bronson, Los Alamos Scientific Laboratory Reports LA-6641-MS (1977).

${ }^{64} \mathrm{~N}$. B. Vargaftik, in Tables on the Thermophysical Properties of Liquids and Gases (Wiley, New York, 1975), p. 7.

${ }^{65}$ H. Shimizu, E. M. Brody, H. K. Mao, and P. M. Bell, Phys. Rev. Lett. 47, 128 (1981).

${ }^{66}$ T. J. Lenosky, J. D. Kress, L. A. Collins, and I. Kwon, Phys. Rev. B 55, R11907 (1997).

${ }^{67}$ W. R. Magro, D. M. Ceperley, C. Pierleoni, and B. Bernu, Phys. Rev. Lett. 76, 1240 (1996)

${ }^{68}$ M. Ross, Phys. Rev. B 58, 669 (1998).

${ }^{69}$ J. van Straaten, R. J. Wijngaarden, and I. F. Silvera, Phys. Rev. Lett. 48, 97 (1982).

${ }^{70}$ J. van Straaten and I. F. Silvera, Phys. Rev. B 37, 1989 (1988).

${ }^{71}$ CRC Handbook of Chemistry and Physics (CRC, Boca Raton, FL, 1994), ed. 75 , pp. 6-20.

${ }^{72}$ D. H. Liebenberg, R. L. Mills, and J. C. Bronson, Phys. Rev. B 18, 4526 (1978).

${ }^{73}$ M. Ross, J. Chem. Phys. 71, 1567 (1979). 